\title{
Erratum to: Using Decomposition Techniques to Determine Cause of Difference in MRIO Databases
}

\author{
Anne Owen
}

\section{Erratum to:}

Chapter 5 in: A. Owen, Techniques for Evaluating the Differences in Multiregional Input-Output Databases, Developments in Input-Output Analysis, DOI 10.1007/978-3-319-51556-4_5

The original version of the book was inadvertently published with a spell error in Chapter 5 title. The spell error was corrected. The erratum chapter and the book have been updated with the change.

The updated original online version for this chapter can be found at http://dx.doi.org/10.1007/978-3-319-51556-4_5

\footnotetext{
A. Owen $(\square)$

University of Leeds, Leeds, UK

e-mail: a.owen@leeds.ac.uk 